\title{
Post-merger electromagnetic emissions from disks perturbed by binary black holes
}

\author{
Matthew Anderson ${ }^{1}$, Luis Lehner ${ }^{2,3,4}$, Miguel Megevand ${ }^{1}$ and David Neilsen ${ }^{5}$ \\ ${ }^{1}$ Department of Physics and Astronomy, \\ Louisiana State University, \\ Baton Rouge, LA 70803-4001 \\ ${ }^{2}$ Perimeter Institute for Theoretical Physics, \\ Waterloo, Ontario N2L 2Y5, Canada \\ ${ }^{3}$ Department of Physics, University of Guelph, \\ Guelph, Ontario N1G 2W1, Canada \\ ${ }^{4}$ CIFAR, Cosmology $\&$ Gravity Program \\ ${ }^{5}$ Department of Physics and Astronomy, \\ Brigham Young University, Provo, UT 84602
}

(Dated: May 29, 2018)

\begin{abstract}
We simulate the possible emission from a disk perturbed by a recoiling super-massive black hole. To this end, we study radiation transfer from the system incorporating bremsstrahlung emission from a Maxwellian plasma and absorption given by Kramer's opacity law modified to incorporate blackbody effects. We employ this model in the radiation transfer integration to compute the luminosity at several frequencies, and compare with previous bremsstrahlung luminosity estimations from a transparent limit (in which the emissivity is integrated over the computational domain and over all frequencies) and with a simple thermal emission model. We find close agreement between the radiation transfer results and the estimated bremsstrahlung luminosity from previous work for electromagnetic signals above $10^{14} \mathrm{~Hz}$. For lower frequencies, we find a self-eclipsing behavior in the disk, resulting in a strong intensity variability connected to the orbital period of the disk.
\end{abstract}

PACS numbers:

\section{INTRODUCTION:}

The near possibility of detecting gravitational waves to study astrophysical systems has spurred efforts to understand such systems theoretically to aid in the detection and interpretation of obtained signals (see, for instance, [1, 2, 3, 4, 5, 6] and references cited therein for further details and examples). Systems that radiate strongly in both electromagnetic and gravitational wave bands are particularly interesting since the combined information will provide unprecedented access to a number of rich phenomena (e.g. [7, 8, 9].) Electromagnetic radiation from compact objects can be (partially or completely) absorbed and scattered by dust and other surrounding material. Gravitational radiation, on the other hand, is generated in the central engine, and these waves do not interact with dust or material in the surrounding environment.

One such system involves the collision of a binary black hole within a circumbinary disk [10, 11, 12]. For a generic black hole binary, the gravitational waves are radiated asymmetrically. As these waves carry energy and momentum, the resultant black hole can have a kick or recoil velocity [13, 14, 15, 16, 17, 18], which in turn will induce shocks in the disk. A second source of perturbations in the disk comes from the loss of gravitational mass with the emission of gravitational radiation, weakening the gravitational potential felt by the disk. These effects, as discussed in [19, 20, 21], can induce possible emissions and some of these have been investigated in part in [20, 21, 22, 23, 24]. These works study the dy- namics of the disks when either mass reduction (due to the energy lost by the system) or recoil velocity (due to the asymmetric flux of gravitational radiation) effects are included and present preliminary estimates of emissions from the system with the goal of understanding possible observations in the electromagnetic spectrum.

In the present work, we further investigate electromagnetic radiation from shocked disks by directly computing the electromagnetic emission using radiation transfer. We build on our earlier work on disks [23], hereafter referred to as Paper I, where we followed the evolution of disks when the central black hole was given different recoil velocities. We use this data for the evolution of disks to calculate the electromagnetic spectrum using radiation transfer. In this method, we assume simple models for the emission and absorption of electromagnetic radiation and solve for the intensities by integrating the radiation transfer equation along geodesics of the spacetime. We examine multiple radiation models to investigate how different choices in the emission models affect the timedependent luminosity. One model assumes only classical thermal emission, and a second model incorporates both bremsstrahlung and thermal emission according to optical depth. Finally, we also compare our results with the simple bremsstrahlung estimate that we obtained in Paper I. 


\section{RADIATION TRANSFER}

Electromagnetic radiation follows geodesics of the spacetime. As the photon is massless, the radiation follows null geodesics, meaning that the proper separation between all points on the geodesic is zero. We calculate the geodesics from the spacetime metric $g_{\alpha \beta}$, and use the convention that Greek indices take the values $\{0,1,2,3\}$. The null geodesics are found by solving the first order system

$$
\begin{aligned}
& \frac{\mathrm{d} x^{\mu}}{\mathrm{d} \lambda}=p^{\mu}, \\
& \frac{\mathrm{d} p_{\mu}}{\mathrm{d} \lambda}=g^{\gamma \beta} \Gamma^{\alpha}{ }_{\mu \gamma} p_{\alpha} p_{\beta},
\end{aligned}
$$

where $\Gamma^{\alpha}{ }_{\mu \gamma}$ are the Christoffel symbols, and $x^{\mu}$ and $p^{\mu}$ are the coordinates and momentum of the photon, respectively. Since we are dealing with null geodesics we parameterize the solution in terms of the affine parameter $\lambda$. We solve the geodesic equations using the standard Runge-Kutta fourth order integration method, numerically integrating the geodesics backwards in time, perpendicularly from a viewing window. This window is set at different locations, in order to provide different viewing angles of the disk.

After obtaining the geodesic paths in the spacetime, we can now solve for the electromagnetic radiation intensity along these paths. The radiation transfer equation for the photon intensity at frequency $\nu$ is 25 ]

$$
\frac{\mathrm{d} I_{\nu}}{\mathrm{d} \lambda}=-p^{\alpha} u_{\alpha}\left[\eta_{0}-\chi_{0} I_{\nu}\right]
$$

where $\chi_{0}$ and $\eta_{0}$ are, respectively, the absorption and emission coefficients in the rest frame with respect to the fluid, and $u_{\alpha}$ is the four-velocity of the fluid. In vacuum regions, the fluid four-velocity corresponds to the four-velocity of coordinate observers. This first order radiation transfer equation has the solution [25]

$$
\begin{aligned}
I_{\nu}(\lambda)= & I_{\nu}\left(\lambda_{0}\right) e^{\int_{\lambda_{0}}^{\lambda} \chi_{0}\left(\lambda^{\prime}, \nu_{0}\right) u_{\alpha} p^{\alpha} d \lambda^{\prime}}- \\
& \int_{\lambda_{0}}^{\lambda} e^{\int_{\lambda^{\prime}}^{\lambda} \chi_{0}\left(\lambda^{\prime \prime}, \nu_{0}\right) u_{\alpha} p^{\alpha} d \lambda^{\prime \prime}} \eta_{0}\left(\lambda^{\prime}, \nu_{0}\right) u_{\alpha} p^{\alpha} d \lambda^{\prime}
\end{aligned}
$$

where $\nu_{0}$ is the photon frequency at emission, and $\lambda_{0}$ indicates the affine parameter value where the geodesic begins. The integration constant $I_{\nu}\left(\lambda_{0}\right)$ takes into account the photon intensity where the geodesic begins. In this work, the geodesics begin far enough behind the disk so that there is no matter to act as a source for emission, and we set $I_{\nu}\left(\lambda_{0}\right)$ to be zero. The fluid variables come from a numerical solution that is specified on a finite difference grid. When integrating Eq. (4), these fluid variables are interpolated both in space and time.
As mentioned above, we employ two different emission models in calculating the spectrum from the kicked disk. The first model assumes that emission from the optically thin region is thermal bremsstrahlung, and the optically thick region is a classical blackbody [26]. The second model uses only thermal emission. Both models use Kramer's opacity law for the absorption. We expect the first model to be more realistic, as the optically thin regions of the disk are expected to radiate primarily with bremsstrahlung, rather than thermal emission, and the second model provides a useful comparison to gauge the importance of the bremsstrahlung emission. We refer to these models as bremsstrahlung-blackbody and thermal emission models, respectively.

The bremsstrahlung-blackbody radiation transfer model uses the standard bremsstrahlung emissivity and a modified form of Kramer's opacity law for the absorption. The bremsstrahlung emissivity is modeled on a Maxwellian plasma [27]

$$
\begin{aligned}
\eta_{0}= & 6.8 \times 10^{-38} Z^{2} n_{e} n_{i} T^{-1 / 2} \\
& \times \bar{g}_{\mathrm{ff}}(\nu, T) e^{-x} \frac{\mathrm{erg}}{\mathrm{s} \mathrm{cm}^{3} \mathrm{~Hz}},
\end{aligned}
$$

where

$$
x \equiv h \nu / k T \text {. }
$$

In these equations $h$ is Planck's constant, $k$ is Boltzmann's constant, $T$ is the temperature of the fluid, $n_{e}$ is the electron number density, $n_{i}$ is the ion number density, $Z=n_{e} / n_{i}$, and $\bar{g}_{\mathrm{ff}}$ is the temperature averaged Gaunt factor for free-free transitions in pure hydrogen. The electron and ion number densities are given by

$$
n_{e}=\frac{\rho}{\mu_{e} m_{p}}, \quad n_{i}=\frac{\rho}{\mu_{i} m_{p}}
$$

where

$$
\mu_{e}=\frac{2}{1+X}, \quad \mu_{i}=\frac{4}{1+3 X}
$$

Here $\rho$ is the rest density of the fluid, $m_{p}$ is the mass of the proton, and $X$ is the relative abundance of hydrogen in the universe, which we set to be $X=0.75$. We use a temperature averaged Gaunt factor, $\bar{g}_{\mathrm{ff}}$, based on 27], where different approximations are given depending on the values of $T$ and $\nu$. To completely specify $\bar{g}_{\mathrm{ff}}$, some criteria must be used to set the transition between approximations valid for $\nu \ll k T / h$ and $\nu \approx k T / h$ in the regime $T(\mathrm{~K}) \gg 3 \times 10^{5} Z^{2}$. We set the transition at the values $[\nu, T]$ for which $\bar{g}_{\mathrm{ff}}$ in the two approximations coincide, giving 


$$
\bar{g}_{\mathrm{ff}}(\nu, T)=\left\{\begin{array}{cc|c}
\frac{\sqrt{3}}{\pi}\left[17.7+\log \left(\frac{T^{3 / 2}}{\nu Z}\right)\right] & \text { if } \quad \nu \leq 10^{9} T^{3 / 2} \\
1 & \text { if } \quad \nu>10^{9} T^{3 / 2} & \text { and } T \leq 3 \times 10^{5} Z^{2} \\
x^{2 / 5} & \text { if } \quad x<1 \\
1 & \text { if } 1 \leq x<29.6 \\
\frac{\sqrt{3}}{\pi} \log (2.2 x) & \text { if } \quad 29.6 \leq x & \text { and } T>3 \times 10^{5} Z^{2}
\end{array} \mid\right. \text { }
$$

where $T$ is in units of Kelvin, and $\nu$ in Hertz. Following [26], we use for the absorption a modified Kramer's opacity law (compare to Eq. 12 below),

$$
\chi_{0}=5 \times 10^{24} \rho^{2} T^{-7 / 2}\left(\frac{1-e^{-x}}{x^{3}}\right) \mathrm{cm}^{-1} .
$$

This modification has the net effect of adding thermal radiation for optically thick regions, while optically thin regions radiate with bremsstrahlung. In optically thick regions, all photons are absorbed and $\mathrm{d} I_{\nu} / \mathrm{d} \lambda \rightarrow 0$, giving the expected blackbody intensity

$$
I_{\nu}=\frac{\eta_{0}}{\chi_{0}} \propto T^{3} x^{3} \frac{e^{-x}}{1-e^{-x}} .
$$

In the second radiation transfer model we consider only the emission of thermal radiation. We use Kramer's opacity law for the absorption

$$
\chi_{0}=5 \times 10^{24} \rho^{2} T^{-7 / 2} \mathrm{~cm}^{-1},
$$

and specify the emissivity using Kirchoff's law [28]

$$
\eta_{0}=\chi_{0} B_{\nu}(T),
$$

where $B_{\nu}(T)$ is given by the Planck law

$$
B_{\nu}(T)=\frac{2 h \nu^{3} / c^{2}}{e^{x}-1} .
$$

Thermal radiation is emitted by matter in thermodynamic equilibrium, while blackbody radiation occurs when the radiation is itself in thermodynamic equilibrium. In optically thick regions $\left(\mathrm{d} I_{\nu} / \mathrm{d} \lambda \rightarrow 0\right)$, we again reduce to the blackbody intensity, $I_{\nu}^{\mathrm{BB}}=B_{\nu}(T)$.

Having specified our emission models, we briefly mention some physical effects not yet contemplated in our analysis. As photons move away from the gravitational sources, their frequencies change due to gravitational redshift. As the redshift varies depending on the location at which the photon is emitted, this leads to a broadening of the emission lines. A simple estimate in a Schwarzschild spacetime shows that gravitational redshift accounts for typical frequency changes of $5 \%$ percent or less if we consider photons being emitted from a minimum distance to the black holes of about $10 R_{\mathrm{S}}$, consistent with the disk in our model. Broadening will occur also due to relativistic Doppler, especially in the inclined views. Considering the fluid velocities in our model, up to about $0.28 c$, we estimate a maximum Doppler shift of about $25 \%$. As the frequency bins used here are much larger than those changes, we do not include the line broadening caused by Doppler or gravitational redshift in our model, and we work only with the original emission frequencies.

Finally, in Paper I we estimated the total bremsstrahlung luminosity by assuming a transparent gas. In this approximation the luminosity is obtained by integrating the bremsstrahlung emissivity, $\epsilon_{B} \propto \rho^{2} T^{1 / 2}$, over the computational domain. We compare the results computed here with this earlier model, and find that this simple estimation works very well for the higher frequencies that we consider.

\section{RESULTS}

In paper I the dynamics of a disk affected by a possible recoil velocity of a newly merged black hole was studied. A salient feature of the analysis presented there is that the recoil influences strongly the disk's behavior as long as it has a component on the orbital plane. This induces shocks in the disk which in turn heat the gas and can lead to emissions with a variability tied to the orbital period at the disk. The results presented in Paper I were obtained assuming the simple ideal fluid equation of state

$$
P=(\Gamma-1) \rho \epsilon,
$$

where $P$ is the fluid pressure, $\epsilon$ is the specific internal energy, and we choose $\Gamma=5 / 3$, considering the gas as being monatomic. We assume that there is no energy transfer from the fluid to the electromagnetic radiation, and 
this equation of state allows for fluid flows, e.g. shocks, that are not isentropic. To calculate the emissivity and absorption coefficients, we obtain the fluid temperature from the ideal fluid law as

$$
T=\frac{\mu m_{\mathrm{p}}}{k} \frac{P}{\rho},
$$

where

$$
\frac{1}{\mu}=\frac{1}{\mu_{e}}+\frac{1}{\mu_{i}},
$$

and $\mu_{e}$ and $\mu_{i}$ are given in Eqs. (8).

The simulations in Paper I correspond to a fixed spacetime background, and thus some variables can be independently rescaled a posteriori. For example, one can rescale the black hole's mass and the disk's density independently, which in turn fixes other quantities such as the luminosity. This scale invariance, however, does not hold for the emission and absorption coefficients in the radiation transfer equation (4), and we set the physical scales in the following manner. We start by setting a central black hole mass $M_{\mathrm{BH}}=1 \times 10^{8} M_{\odot}$, consistent with super-massive black holes at galactic centers. This choice fixes the location of the maximum fluid density $\rho_{\mathrm{m}}$ in our model to $r_{\mathrm{m}} \approx 4.5 \times 10^{9} \mathrm{~km}$, approximately 15 Schwarzschild radii $\left(R_{S}\right)$ from the center of the disk. To estimate a physically reasonable value for the disk's density, we use an alpha-disk model [29]. Note that this model, unlike ours, corresponds to an accreting disk. However, as an estimate we assume that the density in the alpha-disk model will be similar to our disk at the premerger stage, before the inner edge of the disk "freezes" and accretion stops 10, 12]. We set the accretion rate in the alpha disk model to be an Eddington-limited accretion rate of $1 M_{\odot} /$ year. We choose $\alpha=0.1$, a value typical for cataclysmic variables, low-mass X-ray binaries, and black hole transients. Using these parameters in the alpha-disk model we obtain a density $\rho \approx 0.01 \mathrm{~g} / \mathrm{cm}^{3}$ at $r=r_{\mathrm{m}}$. Hence, we scale the disk density so that $\rho_{\mathrm{m}}=0.01 \mathrm{~g} / \mathrm{cm}^{3}$.

As discussed in Paper I, the qualitative behavior for all of the disks that we considered was similar, provided that the recoil velocity has a component orthogonal to the disk's angular momentum. We thus adopt as a representative example the data obtained for a kick perpendicular to the axis of rotation at $3000 \mathrm{~km} / \mathrm{s}$. We place a square-shaped window at three different locations, providing viewing angles of $0^{\circ}, 60^{\circ}$, and $75^{\circ}$ with respect to the disk's rotational axis, while the azimuthal direction is chosen perpendicular to the kick. In all cases the window is located at a distance of $1.05 \times 10^{10} \mathrm{~km}\left(\simeq 35 R_{S}\right)$ away from the black hole (at the edge of the computational domain employed in Paper I). The area of the observation window was $4.41 \times 10^{20} \mathrm{~km}^{2}$ so that the whole of the disk could be analyzed in such window. The number of geodesics used for the window and the number of points used to integrate the radiation transfer equation were selected so that the results did not show appreciable differences upon further increasing them.

We concentrate on photons having initial frequencies from infrared to gamma ray (within the range $10^{12}$ $10^{21} \mathrm{~Hz}$ ) and integrate the radiation transfer equation to the window where we examine the resulting luminosity vs initial frequency. We compare results obtained from the bremsstrahlung-blackbody and thermal models using radiation transfer. We also compare bremsstrahlung luminosity estimated in the transparent limit (Paper I) with the results from radiation transfer. This allows us to examine interesting frequency dependencies not highlighted by the bremsstrahlung luminosity estimate by itself.

For instance, Figures 1 and 2 illustrate the luminosity for the case of soft x-ray photons (initial frequency of $10^{18} \mathrm{~Hz}$ ). Figure 1 shows normalized luminosity curves (with respect to the initial value) obtained via the radiation transfer equation for a window located at $0^{\circ}$ viewing angle (the top view) while Figure 2 shows normalized luminosity curves for different viewing angles. In Figure 1 the results for two radiation models are shown: bremsstrahlung for emissivity and modified Kramer's opacity law for absorption (bremsstrahlung-blackbody), and thermal emissivity with unmodified Kramer's opacity law (thermal). Also included is the normalized bremsstrahlung luminosity from Paper I which was calculated assuming a transparent gas, and integrated over all frequencies (see Eq. 16 of Paper I). The good agreement seen in Figure 1 between the bremsstrahlung-blackbody radiation transfer luminosity curve and the bremsstrahlung luminosity estimate from Paper I indicates that the disk is largely transparent at $10^{18} \mathrm{~Hz}$.

Interestingly this behavior no longer holds for lower frequencies. For instance, Figure 3 illustrates the results obtained in the infrared (initial frequency of $10^{12} \mathrm{~Hz}$ ). At this lower frequency, much of the disk is opaque and a self-eclipsing behavior is increasingly evident as the inclination angle increases. More importantly, after the transient behavior, a strong increase in relative luminosity is observed as shocks significantly affect the dynamics of the disk. Finally, Fig. 4 shows the electromagnetic spectrum calculated from 36 frequencies in the range from $10^{12}-5 \times 10^{21} \mathrm{~Hz}$.

As an illustration of the disk structure observed at different initial frequencies and viewing angles, Fig. 5 and Fig. 6]illustrate the intensity obtained at different times. The color map shows areas of higher intensity emission. Comparing these two figures one can clearly see the differences between a mostly transparent case (Fig. 5), and one with absorption (Fig.6), showing self eclipsing, which supports the oscillations shown in Fig. 3. seen only in the opaque case. 


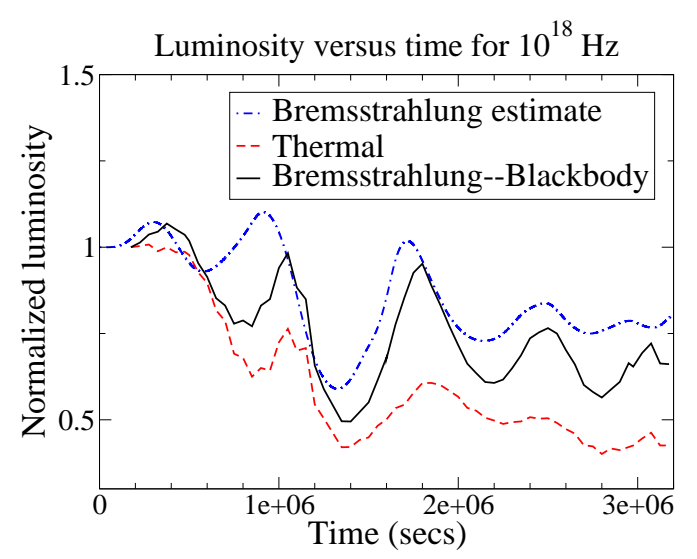

FIG. 1: Normalized luminosity as a function of time using different emission models for a frequency of $10^{18} \mathrm{~Hz}$. The horizontal axis shows time in seconds after the kick. In all cases, the results are normalized with respect to their initial value. The curve labeled "bremsstrahlung estimate" (dash-dot line) is calculated by assuming a transparent gas and integrating $\rho^{2} T^{1 / 2}$ over the computational domain. The curve labeled "thermal" (dashed line) is obtained using the thermal radiation model described in the text for a $0^{\circ}$ viewing angle (top view). The one labeled "bremsstrahlung-blackbody" (solid line) is obtained by using the bremsstrahlung-blackbody radiation model described in the text, also at a $0^{\circ}$ viewing angle.

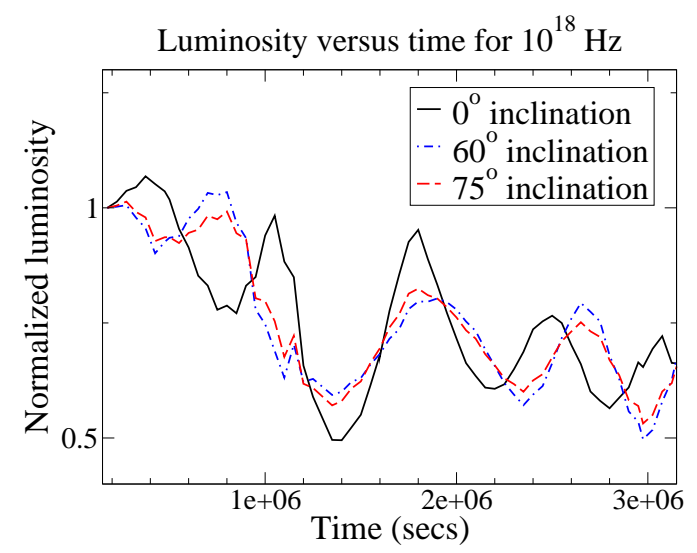

FIG. 2: Normalized bremsstrahlung-blackbody luminosity as a function of time for different viewing angles for a frequency of $10^{18} \mathrm{~Hz}$. The horizontal axis is time in seconds after the kick.

\section{FINAL COMMENTS}

We have analyzed the possible emission of a disk perturbed by a recoiling black hole by directly imaging the disk with radiation transfer. For frequencies above $\sim 10^{13} \mathrm{~Hz}$ our studies reveal a characteristic luminosity variability that could be present in observations of

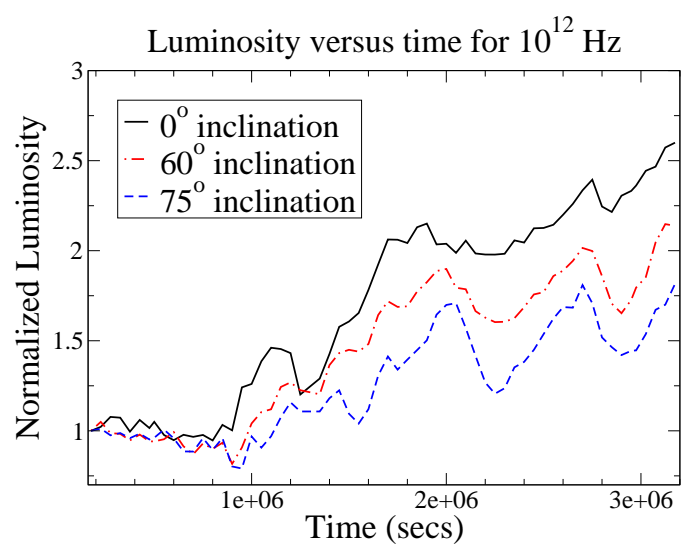

FIG. 3: Normalized luminosity as a function of time for different viewing angles for a frequency of $10^{12} \mathrm{~Hz}$. The luminosity was normalized with respect to its initial value. The horizontal axis labels time in seconds after the kick. At $10^{12} \mathrm{~Hz}$ much of the disk is opaque and exhibits self-eclipsing behavior. The oscillations seen after $t=1 \times 10^{6}$ seconds are tied to the orbital period of the disk and are more pronounced as the viewing angle increases.

this kind of systems. This variability shows the same main features as those in our previous results, in which a simpler model -without radiation transfer- was used to estimate the luminosity.

For frequencies below $\sim 10^{13} \mathrm{~Hz}$ the spectrum matches the Rayleigh-Jeans frequency dependence expected from a blackbody. Furthermore, at these lower frequencies, a self-eclipsing behavior at lower frequencies is clearly seen as the disk orbits around the black hole, so that bright and opaque regions alternatively superpose along the line of sight, resulting in an induced variability tightly tied to the orbital motion and inclination. This effect is less pronounced as higher frequencies are considered and so it is unlikely this feature will be observed.

Nevertheless, a strong overall variability is observed at all frequencies associated with shock heating in sectors of the disk which could be detected provided that the increase in the disk's luminosity prior to the recoil is sufficiently low. This appears as a likely scenario as the binary hollows out the inner regions of the disk [12]. One should be cautious that uncertainties on the details of these disks and emission processes can affect the characteristics of the luminosities obtained. In particular, the work of 24], which employs a different equation of state, displays interesting differences with the ones presented here. However, regardless of particular differences induced by details of the equation of state employed, a common message of all related works [20, 21, 22, 23, 24] is that emissions from circumbinary disks around merging black holes possibly display particular features that could be detected. These features would follow the black hole merger in the time-frame of months and would require a good localization in the sky to follow gravitational 


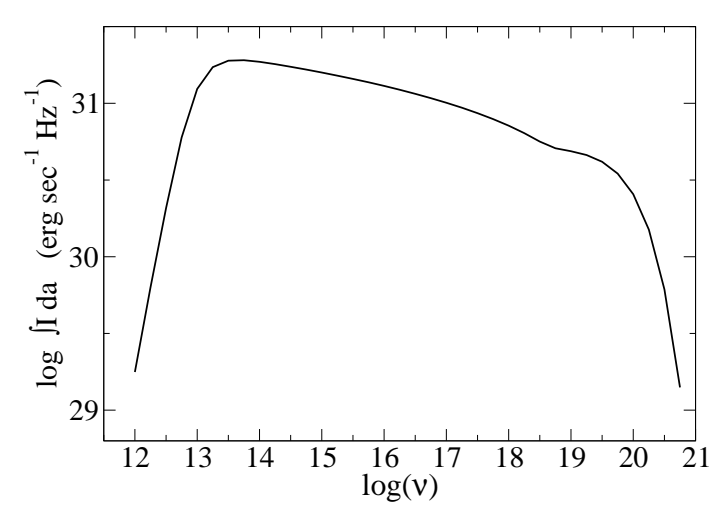

FIG. 4: The electromagnetic spectrum computed $1.25 \times 10^{6}$ seconds after the kick using the "bremsstrahlung-blackbody" radiation model at a $0^{\circ}$ viewing angle. The horizontal axis is the $\log _{10}$ of the frequency. The vertical axis is the $\log _{10}$ of the intensity integrated over the window area. In the lower frequencies of the spectrum $10^{12}-10^{13} \mathrm{~Hz}$ we see the expected Rayleigh-Jeans $\nu^{2}$ frequency dependence. The small kink in the spectrum in between $10^{18}$ and $10^{19} \mathrm{~Hz}$ is due to the behavior of the Gaunt factor in that region. The spectrum was calculated using 36 frequencies, spaced evenly in log space from $10^{12}-5.6 \times 10^{21} \mathrm{~Hz}$. wave observations with these electromagnetic counterparts. As already discussed in the literature (e.g. 8] and references cited therein) LISA's localization will unlikely be able to single out a preferred galaxy and so prospects of detection will be greatly enhanced if pre-cursors or prompt emissions in the electromagnetic wave band are also available [8]. In the case of binary black hole mergers that give rise to the scenario analyzed in this work, such a possibility is just beginning to be analyzed in detail 30, 31, 32, 33, 34.

Acknowledgments: We would like to thank J. Frank, C. Palenzuela, E. Hirschmann, P. Motl, S. Liebling, J. Staff and J. Tohline for stimulating discussions. This work was supported by the NSF grants PHY-0803629 to LSU, PHY-0803615 and CCF-0832966 to BYU. Computations were done at BYU, LONI, LSU, and TeraGrid.
[1] B. Aylott et al., Class. Quant. Grav. 26, 114008 (2009), 0905.4227.

[2] B. Aylott et al., Class. Quant. Grav. 26, 165008 (2009), 0901.4399

[3] D. Shoemaker, B. Vaishnav, I. Hinder, and F. Herrmann, Class. Quant. Grav. 25, 114047 (2008), 0802.4427.

[4] T. Baumgarte et al., Phys. Rev. D77, 084009 (2008), gr-qc/0612100.

[5] Y. Pan et al., Phys. Rev. D77, 024014 (2008), 0704.1964.

[6] P. Ajith et al., Phys. Rev. D77, 104017 (2008), 0710.2335 .

[7] Z. Haiman, B. Kocsis, K. Menou, Z. Lippai, and Z. Frei (2008), arXiv:0811.1920 [astro-ph].

[8] J. S. Bloom et al. (2009), arXiv:0902.1527 [astro-ph.CO].

[9] L. Blecha and A. Loeb (2008), 0805.1420.

[10] P. J. Armitage and P. Natarajan, Ap. J. Letters 567, L9 (2002), arXiv:astro-ph/0201318.

[11] F. K. Liu, X.-B. Wu, and S. L. Cao, MNRAS 340, 411 (2003), arXiv:astro-ph/0310045.

[12] M. Milosavljevic and E. S. Phinney, Astrophys. J. 622, L93 (2005), arXiv:astro-ph/0410343.

[13] J. G. Baker, W. D. Boggs, J. Centrella, B. J. Kelly, S. T. McWilliams, M. C. Miller, and J. R. van Meter, Astrophys. J. 668, 1140 (2007), arXiv:astro-ph/0702390.

[14] M. Campanelli, C. Lousto, Y. Zlochower, and D. Merritt, Ap. J. Letters 659, L5 (2007), arXiv:gr-qc/0701164.

[15] J. A. González, U. Sperhake, B. Brügmann, M. Hannam, and S. Husa, Physical Review Letters 98, 091101 (2007), arXiv:gr-qc/0610154.

[16] F. Herrmann, I. Hinder, D. Shoemaker, and P. Laguna,
Classical and Quantum Gravity 24, 33 (2007), arXiv:grqc/0601026.

[17] W. Tichy and P. Marronetti, Phys. Rev. D 76, 061502 (2007), arXiv:gr-qc/0703075.

[18] M. Koppitz, D. Pollney, C. Reisswig, L. Rezzolla, J. Thornburg, P. Diener, and E. Schnetter, Physical Review Letters 99, 041102 (2007), arXiv:gr-qc/0701163.

[19] J. D. Schnittman and J. H. Krolik, Astrophys. J. 684, 835 (2008), arXiv:0802.3556.

[20] Z. Lippai, Z. Frei, and Z. Haiman, Ap. J. Letters 676, L5 (2008), arXiv:0801.0739.

[21] G. A. Shields and E. W. Bonning, Astrophys. J. 682, 758 (2008), arXiv:0802.3873.

[22] S. M. O’Neill, M. C. Miller, T. Bogdanovic, C. S. Reynolds, and J. Schnittman, ArXiv e-prints (2008), arXiv:0812.4874.

[23] M. Megevand, M. Anderson, J. Frank, E. W. Hirschmann, L. Lehner, S. L. Liebling, P. M. Motl, and D. Neilsen, ArXiv e-prints (2009), 0905.3390.

[24] L. R. Corrales, Z. Haiman, and A. MacFadyen (2009), 0910.0014.

[25] S. V. Fuerst and K. Wu, A\&A 424, 733 (2004), arXiv:astro-ph/0406401.

[26] J. D. Schnittman, J. H. Krolik, and J. F. Hawley, Astrophys. J. 651, 1031 (2006), astro-ph:0606615.

[27] W. Tucker, Radiation processes in astrophysics (1975).

[28] G. B. Rybicki and A. P. Lightman, Radiative Processes in Astrophysics (John Wiley \& Sons, New York, 1979).

[29] N. I. Shakura and R. A. Sunyaev, A\&A 24, 337 (1973).

[30] C. Palenzuela, M. Anderson, L. Lehner, S. L. Liebling, 
and D. Neilsen, Phys. Rev. Lett. 103, 081101 (2009), 0905.1121.

[31] P. Chang, L. Strubbe, K. Menou, and E. Quataert, Private Communication (2009).

[32] J. R. van Meter et al. (2009), 0908.0023.
[33] C. Palenzuela, L. Lehner, and S. Yoshida, in preparation (2009).

[34] P. Mosta, C. Palenzuela, L. Rezolla, L. Lehner, S. Yoshida, and D. Pollney, in preparation (2009). 

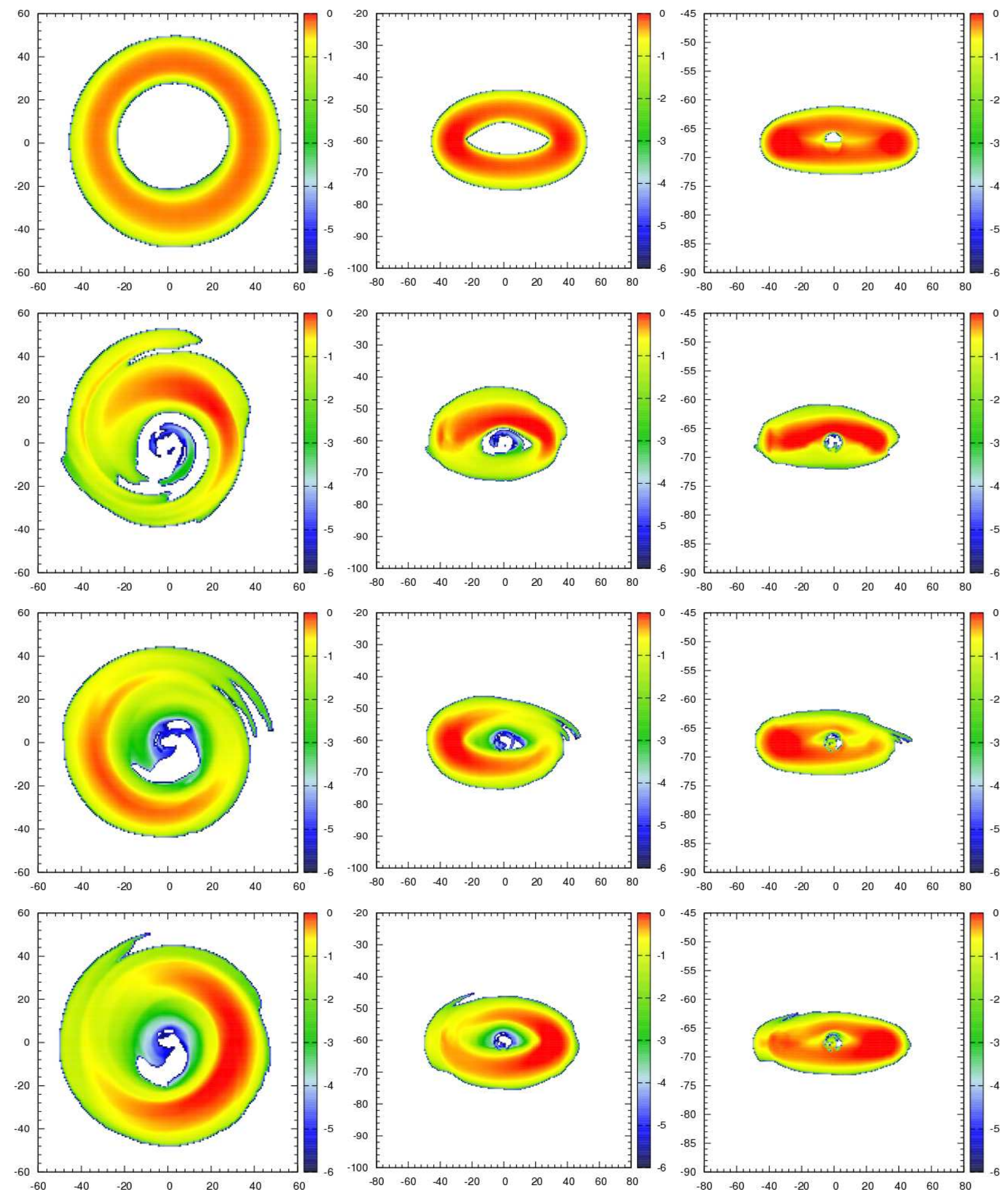

FIG. 5: The intensity for $10^{18} \mathrm{~Hz}$ shown, from top to bottom, at $t=1.75 \times 10^{5}, 1.25 \times 10^{6}, 1.5 \times 10^{6}$, and $1.75 \times 10^{6}$ seconds after the kick using the "bremsstrahlung-blackbody" radiation model. The colormap is a log scale; The intensity for all the images has been normalized using the maximum intensity found at $t=1.25 \times 10^{6}$ seconds after the kick. The images on the left/center/right columns correspond to viewing angles of $0^{\circ} / 60^{\circ} / 75^{\circ}$ respectively. The number of geodesics used was 40,000 . The axis scale of the images is in units of mass of the black hole. 

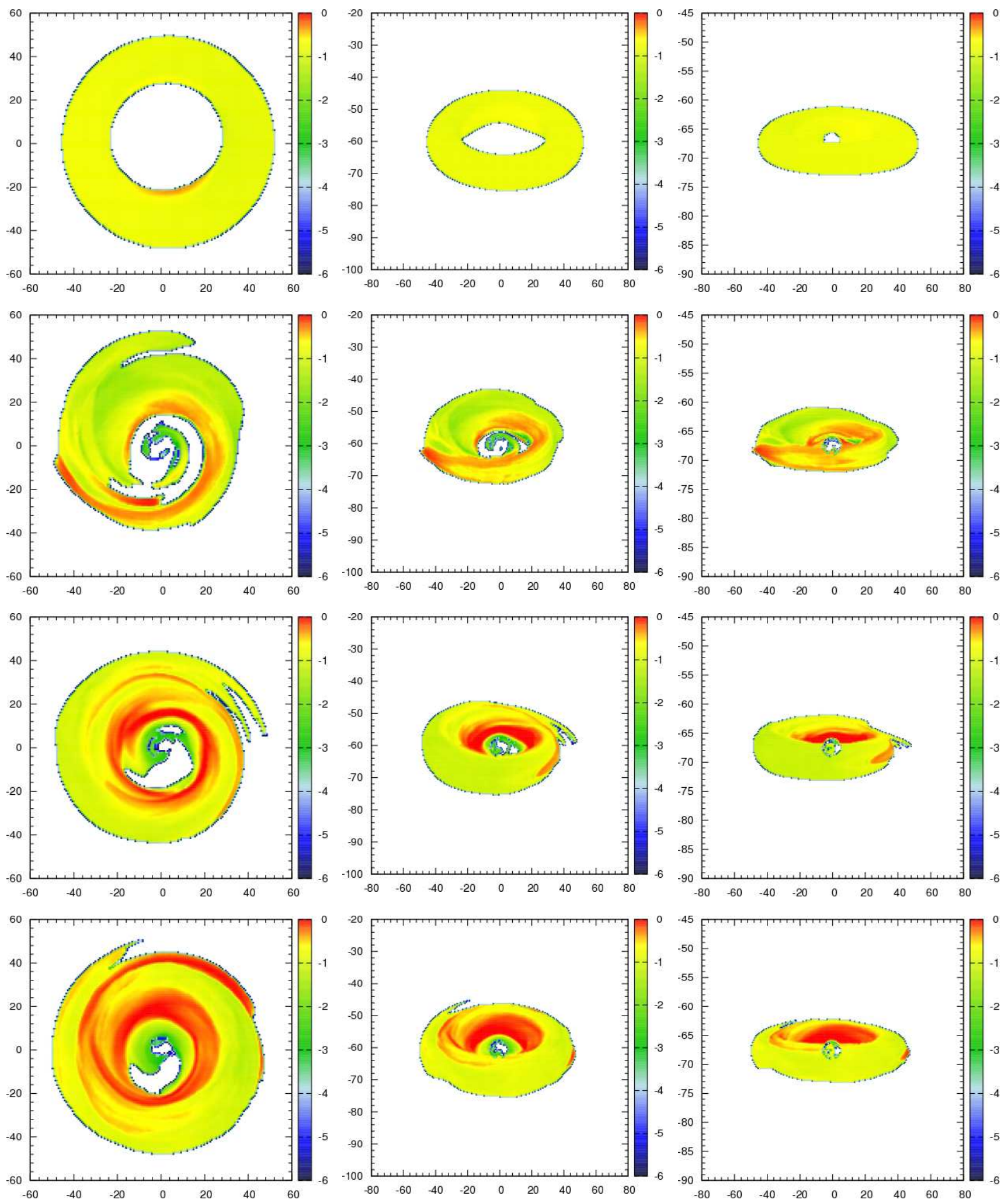

FIG. 6: The intensity for $10^{12} \mathrm{~Hz}$ at $t=1.75 \times 10^{5}, 1.25 \times 10^{6}, 1.5 \times 10^{6}$, and $1.75 \times 10^{6}$ seconds after the kick using the "bremsstrahlung-blackbody" radiation model. The colormap is a log scale; The intensity for all the images has been normalized using the maximum intensity found at $t=1.25 \times 10^{6}$ seconds after the kick. The images on the left/center/right columns correspond to viewing angles of $0^{\circ} / 60^{\circ} / 75^{\circ}$ respectively. The number of geodesics used was 40,000 . The axis scale of the images is in units of mass of the black hole. 